\title{
Pedestal conditions for small ELM regimes in tokamaks
}

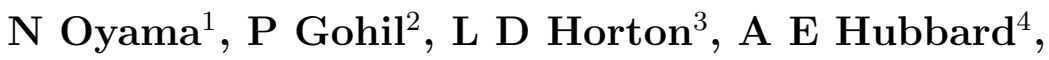 \\ J W Hughes ${ }^{4}$, Y Kamada ${ }^{1}$, K Kamiya ${ }^{1}$, A Leonard $^{2}$, A Loarte ${ }^{5}$, \\ R Maingi ${ }^{6}$, G Saibene ${ }^{5}$, R Sartori ${ }^{5}$, J K Stober $^{3}$, W Suttrop ${ }^{3}$, \\ H Urano ${ }^{1}$, P West $^{2}$ and the ITPA Pedestal Topical Group \\ 1 JAEA-Naka, 801-1 Muko-yama, Naka, Ibaraki 311-0193, Japan \\ ${ }^{2}$ General Atomics, PO Box 85608, San Diego, CA 92186-5608, USA \\ 3 Max-Planck-Institut für Plasmaphysik, EURATOM, Garching, Germany \\ ${ }^{4}$ MIT Plasma Science and Fusion Center, Cambridge, MA 02139, USA \\ ${ }^{5}$ EFDA Close Support Unit-Garching, 2 Boltzmannstrasse, Garching, Germany \\ ${ }^{6}$ Oak Ridge National Laboratory, PO Box 2009, Oak Ridge, TN 37831, USA \\ E-mail: oyama.naoyuki@jaea.go.jp
}

\begin{abstract}
Several small/no ELM regimes such as EDA, grassy ELM, HRS, QHmode, type II and V ELMs with good confinement properties have been obtained in Alcator C-Mod, ASDEX-Upgrade, DIII-D, JET, JFT-2M, JT-60U and NSTX. All these regimes show considerable reduction of instantaneous ELM heat load onto divertor target plates in contrast to conventional type I ELM, and ELM energy losses are evaluated as less than $5 \%$ of the pedestal stored energy. These small/no ELM regimes are summarized and widely categorized by their pedestal conditions in terms of the operational space in non-dimensional pedestal parameters and requirement of plasma shape/configuration. The characteristics of edge fluctuations and activities of ideal MHD stability leading to small/no ELMs are also summarized.
\end{abstract}

\section{Introduction}

H-mode plasmas with type I ELMs are the reference operating scenario for $\mathrm{Q}=10$ inductive operations in ITER. Although there is confidence that the regime can be accessed with sufficient performance, the ability to produce H-mode plasmas with small ELMs is an important step towards extending the lifetime of the divertor target plates in ITER $[1,2]$. An acceptable lifetime of the divertor target plates requires a tolerable ELM energy loss of $<6$ MJ per ELM, which would correspond to about $6 \%$ of the pedestal stored energy in ITER. Another issue of large ELMs is the compatibility with an internal transport barrier (ITB), which is essential for advanced operational scenarios $[3,4]$. Because of these requirements and motivations, extensive studies to establish alternative small/no ELM regimes such as EDA, grassy ELM, HRS, QH-mode, type II and V ELMs, have been carried out in Alcator C-Mod (C-Mod), ASDEX Upgrade (AUG), DIII-D, JET, JFT-2M, JT-60U and NSTX. 
In order to compare the pedestal conditions in these many regimes, they have been categorized into 4 main groups (grassy ELM regime, type II ELM regime, QHmode regime and high $\nu_{e}^{*}$ regime) in terms of ELM energy loss and pedestal electron collisionality $\nu_{e}^{*}$, which plays a significant role in pedestal stability through modification of the edge bootstrap current. Moreover, ITER will have a low collisionality pedestal. Here, $\nu_{e}^{*}$ in this paper is adopted following the formula reported in [5],

$$
\nu_{e}^{*}=6.921 \times 10^{-18} \frac{R q_{95} n_{e} Z_{e f f} \ln \Lambda_{e}}{\varepsilon^{3 / 2} T_{e}^{2}}
$$

where $R, q_{95}$ and $Z_{\text {eff }}$ are the major radius in $\mathrm{m}$ and the safety factor at $95 \%$ of flux surface and effective ion charge, $n_{e}$ and $T_{e}$ are electron density in $m^{-3}$ and temperature in $\mathrm{eV}$ evaluated at the top of pedestal, $\ln \Lambda_{e}$ is the Coulomb logarithm defined by $\ln \Lambda_{e}=31.3-\ln \left(\sqrt{n_{e}} / T_{e}\right)$.

Using the above categorization, the operational space in dimensional and nondimensional pedestal parameters are summarized and compared with each other together with the effect of plasma shape in sections 2-5. Characteristics of edge fluctuations observed in some small/no ELM regimes are reviewed in Section 6. Section 7 presents a brief summary of ideal MHD stability studies aimed to understand the suppression mechanism of large ELMs. Finally Section 8 discusses some issues of these small/no ELM regimes concerning the applicability to ITER plasmas.

\section{High $n_{e}$ type II ELM regime}

Type II ELMs with small ELM amplitude were found in DIII-D with increasing plasma elongation $(\kappa>1.8)$ at high safety factor $\left(q_{95} \sim 7\right)$ and with high triangularity $(\delta \sim 0.5)[6]$. Similar small ELMs have been observed on AUG and JET in highly shaped plasmas at high density [7, 8]. Since type II ELMs do not result in detectable stored energy drop or heat flux at the divertor target, the main loss mechanism in the type II ELM regime has yet to be understood. In JET, no detectable reduction in electron temperature at the pedestal is observed. Therefore, an increase of quasi-continuous losses due to the existence of edge MHD fluctuations is considered as an important characteristic, and therefore enhanced edge MHD fluctuations are one of the criteria to identify the type II ELM regime in AUG and JET. In spite of the enhancement of the inter ELM losses in type I+II regime in JET, the global plasma confinement can be kept as high as the type I ELM regime.

Achieved pedestal pressure in the type II ELM regime is comparable to the usual type I ELM regime as shown in Figs. 1(a) and 1(b) in spite of the existence of edge fluctuations. Moreover, higher pedestal pressure can be obtained in JET. Because of a requirement of high density, the edge collisionality remained at moderate values $\left(\nu_{e}^{*}>0.8\right)$. It should be noted that a narrow operational window in density $\left(0.85<\bar{n}_{e} / n_{G W}<0.95\right)$ is observed in AUG [7]. Figure 2 shows the comparison of the nondimensional operational regime in $\beta_{p}-\nu_{e}^{*}$ space. It suggests that there is not a requirement large of poloidal beta $\beta_{p}$ in contrast to the grassy ELM regime as discussed in the next 

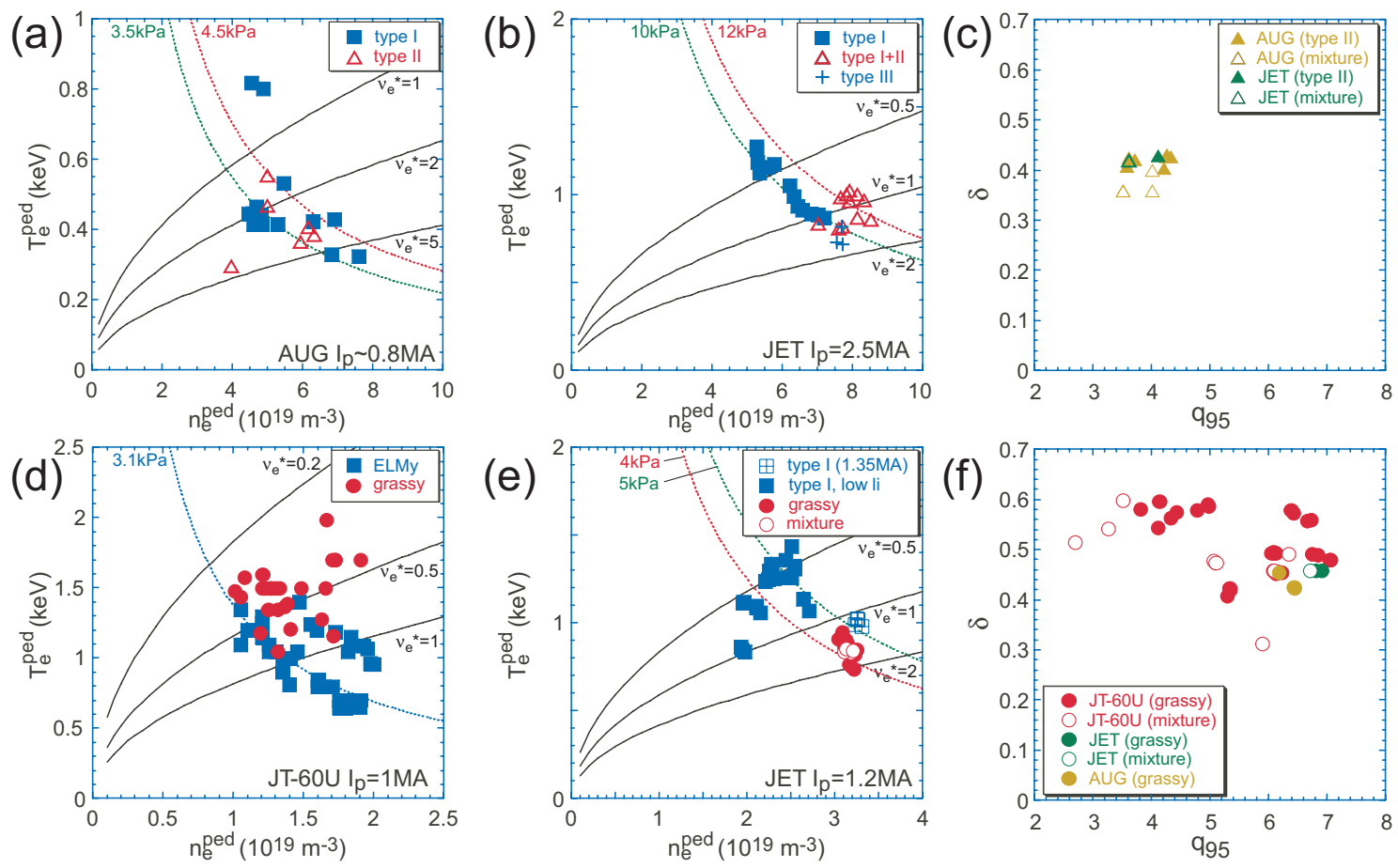

Figure 1. (a), (b) pedestal operational points in type II ELM regime on AUG and JET, respectively. (c), (f) operational regime in $q_{95}-\delta$ space for the type II ELM and grassy ELM regimes, respectively. (d), (e) pedestal operational points in the grassy ELM regime on JT-60U and JET, respectively. All n-T operational diagrams shown in this figure are plotted with selected data with fixed plasma current. Dotted (solid) lines in $\mathrm{n}-\mathrm{T}$ operational diagrams show the line of constant pressure (constant $\nu_{e}^{*}$ ). "Mixture" means that type I+II regime in (c) and type I+grassy ELM in (e) and (f).

section. On the other hand, a quasi-double null (QDN) configuration $\left(\Delta_{\text {sep }} \leq 0.01 \mathrm{~m}\right)$ is required in AUG, where typical operational value is $\delta>0.4$ as shown in Fig. 1(c).

\section{Grassy ELM regime}

The grassy ELM regime was found in JT-60U as another small ELM regime at lower $\nu_{e}^{*}$ in high $\beta_{p}$ plasmas with simultaneously high $q_{95}$ and high $\delta$ [9]. The grassy ELM regime can be extended toward $q_{95}<4$ in JT-60U by using higher $\delta \sim 0.6$ as shown in Fig. 1(f)[10]. In recent experiments on JET and AUG, grassy-like ELMs were also observed following the grassy ELM prescription with high $\beta_{p}$ plasmas $\left(\beta_{p}>1.7\right)$ at high $q_{95}\left(q_{95} \sim 7\right)$ and high triangularity $(\delta>0.4)[11,12]$. As can be seen in Fig. 2, grassy ELMs can be obtained at low collisionality of $\sim 0.3$ in JT-60U. Nevertheless, achieved $\nu_{e}^{*}$ in grassy ELM plasmas was comparable to type II ELM plasmas in JET. It is noted that no significant edge fluctuations related to enhanced losses were observed in any devices with grassy ELMs.

As can be seen in Fig. 1(d), pedestal pressure in the grassy ELM regime can be higher than the usual type I ELM regime thanks to the positive feedback between 


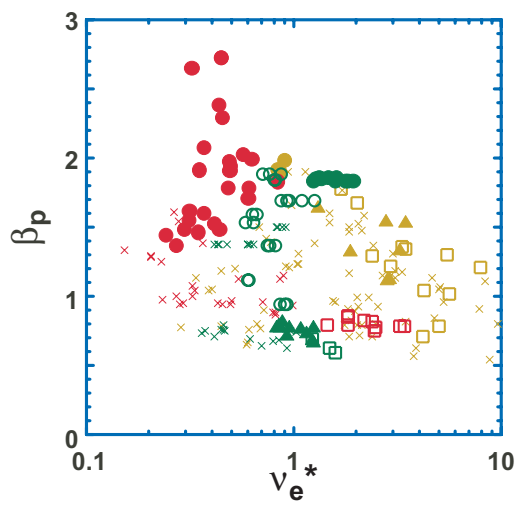

$$
\begin{array}{ll}
\times & \text { AUG (type I) } \\
\triangle & \text { AUG (type II) } \\
\square & \text { AUG (type III) } \\
& \text { AUG (grassy) } \\
& \text { JT-60U (grassy) } \\
\times & \text { JT-60U (type I) } \\
\square \text { JT-60U (type III) } \\
- \text { JET (grassy) } \\
\times \text { JET (type I) } \\
\bigcirc \text { JET (type I, low li) } \\
\wedge \text { JET (type II) } \\
\square \text { JET (type III) } \\
\hline
\end{array}
$$

Figure 2. Operational space in $\nu_{e}^{*}$ v.s. $q_{95}$ for small/no ELM regimes and type I/III ELM regime. Different color shows different devices.

pedestal and core parameters in high $\delta$ plasmas with an internal transport barrier on JT-60U [10]. Grassy ELM plasmas in JET also show similar pedestal pressure to the usual type I ELM plasma as shown in Fig. 1(e). The main loss channel during grassy ELM phase has been investigated in JT-60U and JET [13, 11]. In both devices, a narrow radial extent of the collapse of the temperature pedestal is observed such that the ELM size becomes smaller than type I ELMs without enhancement of inter ELM loss as observed in the type II ELM regime. From this view point, it is considered that grassy ELMs and type II ELMs are different regimes in JET. In contrast to this, grassy ELMs and type II ELMs in AUG have apparent differences in $q_{95}$ and MHD activity and similarities in the requirement of a QDN configuration and higher $\beta_{p}$ operation. Therefore, dedicated experiments are planned to determine whether type II ELMs and grassy ELMs are the same regime or not in AUG. Actually this shows that we do not have a clear understanding of what these ELM regimes are, since we are not yet away from a "device-dependent" phenomenology.

It has been known that there are some important parameters to enter the grassy ELM regime, higher $\beta_{p}$, higher $\delta$ and higher $q_{95}$ [9]. However, these parameters are not sufficient conditions to enter the grassy ELM regime. For instance, higher $\beta_{p}$ seems to be a necessary but not sufficient condition for JET as shown in Fig. 2, and therefore higher internal inductance $l_{i}$ as well as higher $\beta_{p}$ seems to be important in JET [11]. On the other hand, grassy ELMs are obtained in JT-60U at low $l_{i}\left(0.64<l_{i}<0.85\right)$, while some amount of counter toroidal rotation (or corresponding $E_{r}$ profile) seems to be required [13]. These differences in the operational regime seem to be linked to the sensitivity of the edge stability to $\beta_{p}$ and edge current [14] and the relation between $l_{i}$ and edge current profile in both devices should be checked.

\section{QH-mode regime}

QH-mode (quiescent H-mode) regimes, where similar confinement levels as standard ELMing H-mode can be sustained without any ELMs, were originally observed in DIII- 
$\mathrm{D}$ [15], and then also produced in AUG, JT-60U and JET [16, 17, 18]. In these plasmas, counter-NBI (NB injection opposite to the plasma current) and a large clearance between the plasma separatrix and the first wall are required to obtain the QH phase. Edge Harmonic Oscillations (EHOs), which are considered to enhance the particle fluxes, were usually observed near the separatrix at the onset of the QH phase in all devices $[15,16,19,13]$. Edge pressure in QH-mode plasma on JT-60U seems to be lower than that in ELMing plasma as shown in Fig. 3(b), while QH-mode plasma in DIII-D and AUG can sustain similar pedestal pressure.

One of the key parameters for QH-mode operation is lower density, so that effective divertor pumping is usually applied to QH-mode plasmas. When the plasma density increases by gas puffing during the QH-mode phase, type I ELMs appear at a certain density level. A trajectory in pedestal parameters during a density scan is shown in Fig. 3(b). In JT-60U, the pedestal density increases while keeping the same pedestal temperature, so that the edge pressure increases together with density. Then, type I ELMs appear when the pedestal pressure reaches a certain level. The exact boundary has not been established as a function of the other plasma parameters so far. However, a higher $\delta$ plasma has a possibility to increase the critical density as shown in Fig. 3(a).

A relation between characteristics of the EHO and $q_{95}$ has been investigated. A change in the dominant mode number by changing $q$ was observed in AUG [20]. Nevertheless, it seems that there is no preferred value of $q_{95}$ in a range shown in Fig. $3(\mathrm{c})$. Therefore, resonant conditions of edge/pedestal plasma to a certain $q$ surface is not a required condition of $\mathrm{QH}$-mode.

The mechanism of the stabilization of large ELMs is still one of the open questions. One possible explanation is that the pedestal pressure gradient is reduced below the stability limit due to the onset of the EHO. Although such reduction of edge pressure has been observed in JT-60U, edge stability analysis should be performed to check this hypothesis. Another possibility is stabilization by large rotation or electric field shear. In fact, a quite different $E_{r}$ structure was observed near the edge in DIII-D and AUG
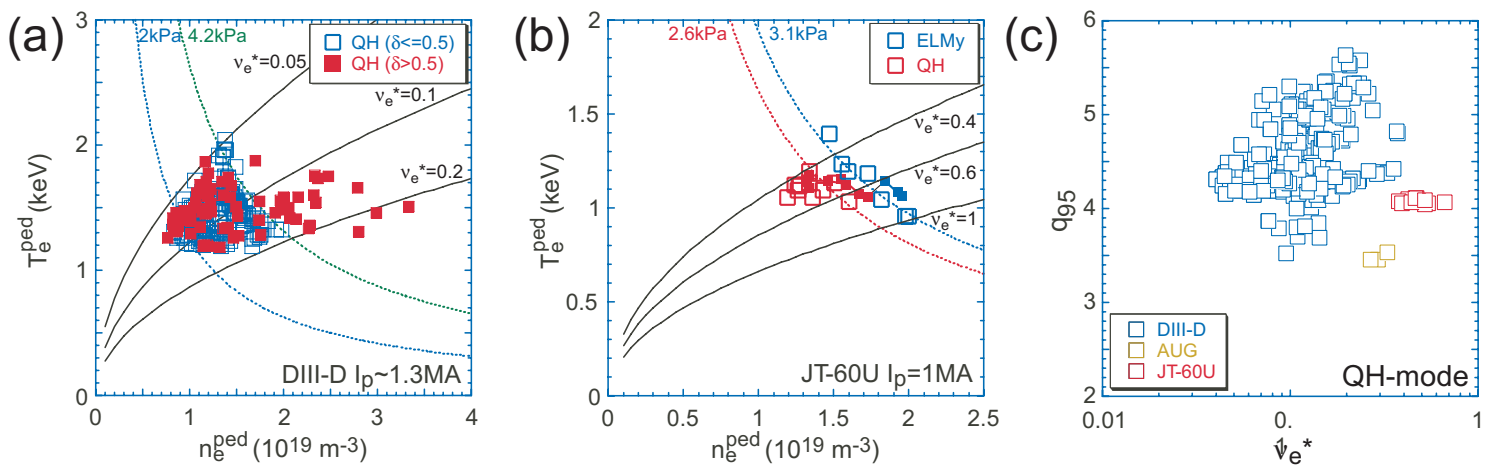

Figure 3. (a), (b) pedestal operational points in QH-mode regime in DIII-D and JT-60U, respectively. Solid and small symbols in (b) show the time evolution of $p_{\text {ped }}$ during a density scan. (c) Comparison of operational regimes in $q_{95}$ versus $\nu_{e}^{*}$. 
during the QH-mode phase [15, 21]. The role of counter-NBI has not been understood yet. Effects of fast ion losses due to different orbit of fast ions are addressed in $[22,18]$ However, partial QH-mode phases were observed in JT-60U during co- and balancedNB injection phase [13]. Further experiment may clarify the requirements to enter the QH-mode.

\section{High $\nu_{e}^{*}$ regime}

Some small/no ELM regimes are usually observed in high recycling and high collisionality condition. The EDA (enhanced $D_{\alpha}$ ) H-mode was found in C-Mod after boronization of the first wall [23]. A similar regime, the so-called HRS (high recycling steady) H-mode, has been observed in JFT-2M [24] after boronization also. Both regimes are characterized by an enhanced $D_{\alpha}$ signal as a consequence of particle losses due to edge MHD and density fluctuations, such that a steady H-mode can be sustained. Recently, another small ELM regime was found in NSTX containing the so-called type V ELMs, where small ELMs can be seen on an enhanced $D_{\alpha}$ signal, similar to the EDA/HRS regimes, in spite of the absence of edge fluctuations (except for an $n=1$ precursor) [25].
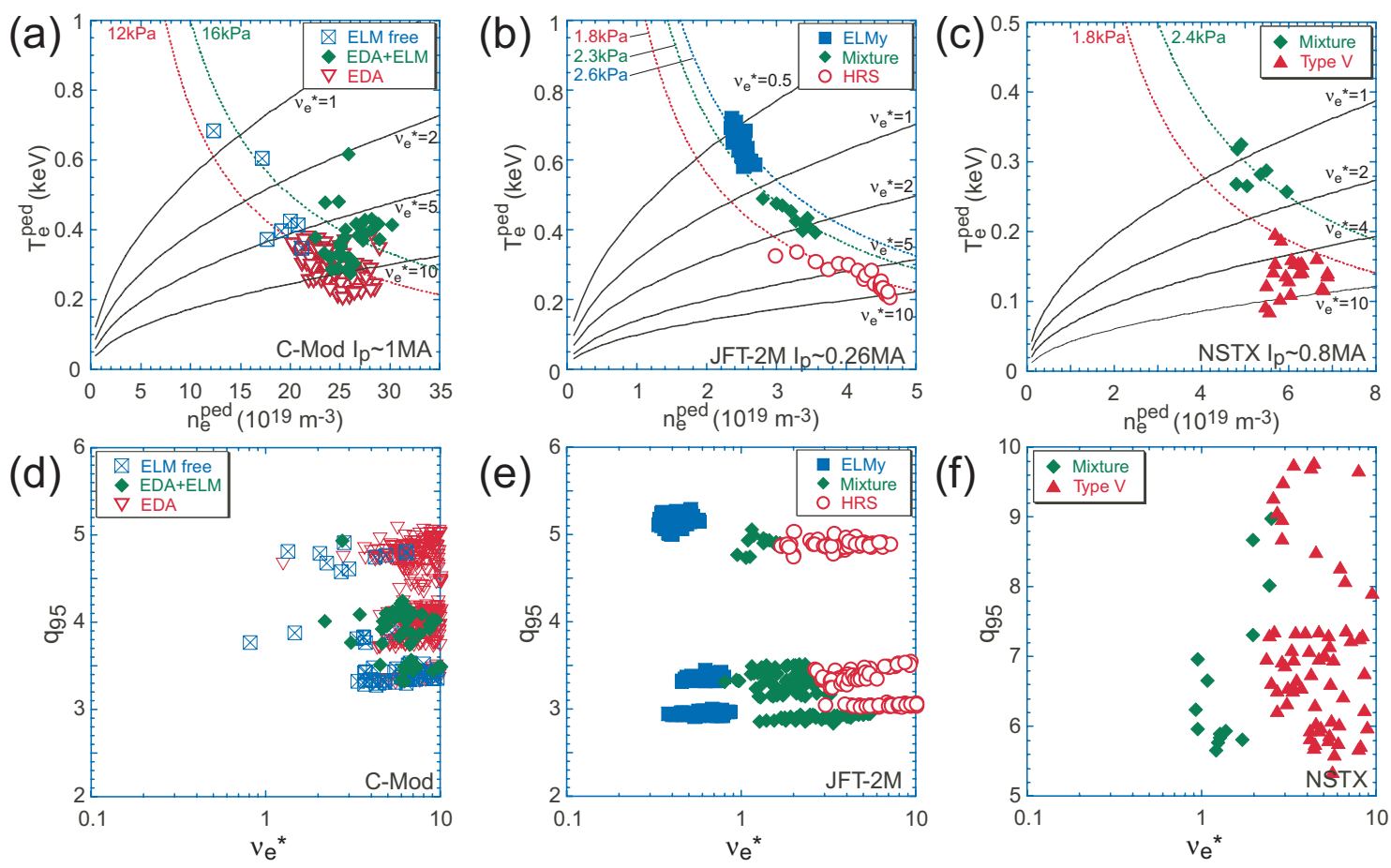

Figure 4. (a)-(c) Comparison of pedestal operational points among EDA, HRS and type V ELM regimes at fixed plasma current. $q_{95}$ shown in (a)-(c) are selected $q_{95} \sim 4.0$ for C-Mod, $q_{95} \sim 3.4$ for JFT-2M and $q_{95} \sim 6$ for NSTX, respectively. The corresponding electron collisionality $\nu_{e}^{*}$ and constant pedestal pressure are shown by solid and dotted lines in (b) and (c), respectively. (d)-(f) Comparison of operational regimes in $q_{95}$ versus $\nu_{e}^{*}$. In these figures, a representative $Z_{\text {eff }}$ value of 2 is used for all devices. 
Figure 4 shows the comparison of pedestal operational points among EDA, HRS and type V ELM regimes. As can be seen in Fig. 4(a)-(c), the pedestal pressure in these small/no ELM regimes generally appear in a smaller region than ELMy/Mixture regimes. Moreover, the pedestal pressure seems to be limited to a certain level, as most clearly observed in Fig. 4(b). Gradual reduction in pedestal pressure in HRS H-mode seems to be related to the mode amplitude of edge fluctuations [26]. At higher pressure in EDA plasmas, small ELMs can be seen on the top of EDA as indicated by EDA+ELM in Fig. 4(a). Because of the enhanced recycling, pedestal densities in these regimes are generally higher than ELMy or ELM-free phases, so that the typical operation point in the edge collisionality appears in the higher regime, $\nu_{e}^{*}>2$, as shown by solid lines in Fig. 4(a)-(c). Effects of the safety factor on the operational space in the collisionality are compared in Fig. 4(d)-(f). In all regimes, the mixture regime of ELMy and small/no ELM regimes appeared at $\nu_{e}^{*} \geq 1$. At mixture phase in type V ELM regime, large ELMs were observed between Type V ELMs when $\beta_{N} \geq 5$ [27]. Effects of plasma shape have been investigated in $q_{95}-\delta$ space in C-Mod and JFT-2M $[28,29]$. In both regimes, higher $\delta$ is preferable for entering EDA/HRS regimes and/or edge fluctuations.

From the view point of the operational regime, observed $\nu_{e}^{*}$ between type II ELMs

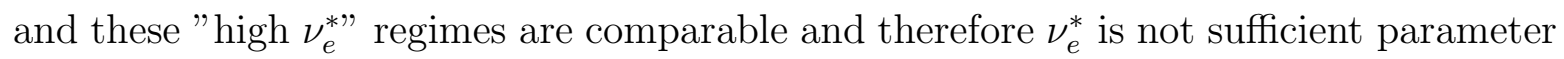
to distinguish among these small/no ELM regimes. One remarkable difference is the level of recycling. The recycling level does not change much at the transition from type I ELMs to type II ELMs, while enhancement of the recycling in EDA, HRS and type V regimes is quite clear. The difference in edge fluctuations observed in these regimes is discussed in the next section.

\section{Edge Fluctuations}

As described in previous sections, the existence of edge fluctuations seems to be important to obtain some small/no ELM regimes such as EDA, HRS, QH-mode and type II ELM regimes. Some important characteristics of edge fluctuations observed in these regimes are summarized in table 1 . The frequency range of edge fluctuations with fundamental mode number in QH-mode is similar in all devices, $f \sim 10 \mathrm{kHz}$, and their typical characteristics of many harmonics corresponding to higher toroidal mode number is also comparable. Broadband characteristics as well as the frequency range (a few tens of $\mathrm{kHz}$ ) of edge fluctuations in type II ELM regimes are similar between AUG and JET, although the dominant mode number is different. Edge fluctuations observed in similar high collisionality regimes, EDA in C-Mod and HRS in JFT-2M, show a different frequency range and mode number, whereas the global nature of these modes are quite similar. Since characteristics of these edge fluctuations can be changed by edge plasma parameters, some inter-machine experiments have been performed recently using the dimensionless identity technique.

Three inter-machine experiments have been performed to reproduce the EDA regime in DIII-D, JET and JFT-2M [30, 12, 31]. Edge fluctuations in a DIII-D 
Table 1. Characteristics of edge fluctuations observed in small/no ELM regimes.

\begin{tabular}{|c|c|c|c|}
\hline $\begin{array}{l}\text { Device } \\
\text { (type) }\end{array}$ & Frequency & $\begin{array}{l}\text { mode number/ } \\
\text { wave number }\end{array}$ & $\begin{array}{c}\text { Fluctuating Parameter } \\
\text { (location) }\end{array}$ \\
\hline \multirow{2}{*}{$\begin{array}{c}\text { DIII-D } \\
(\mathrm{QH})\end{array}$} & $6-10 \mathrm{kHz}$ & $n=1 \sim 11$ & $\overline{B_{\theta}, \tilde{T}, \tilde{n} \text { (edge and SOL) }}$ \\
\hline & (fundamental, EHO) & $m \sim 5\left(k_{\theta} \sim 0.01 \mathrm{~cm}^{-1}\right)$ & $\tilde{I}_{s}(\mathrm{SOL}$ and $\mathrm{DIV})$ \\
\hline \multirow{3}{*}{$\begin{array}{l}\text { AUG } \\
(\mathrm{QH})\end{array}$} & $\approx 10 \mathrm{kHz}$ & $n=1 \sim 11$ & $\widetilde{B_{\theta}, \tilde{T}, \tilde{n}, I_{S X} \text { (edge) }}$ \\
\hline & (fundamental, EHO) & & \\
\hline & $300-450 \mathrm{kHz}(\mathrm{HFO})$ & $n=5, m=6$ & $\bar{B}_{\theta}$ \\
\hline \multirow{2}{*}{$\begin{array}{c}\text { JT-60U } \\
(\mathrm{QH})\end{array}$} & $4-10 \mathrm{kHz}, f_{E H O} \propto V_{T}+C$ & $k_{r} \sim 0.3 \mathrm{~cm}^{-1}$ & $\tilde{T}, \tilde{n}$ (edge) \\
\hline & (fundamental, EHO) & & $\tilde{I}_{s}(\mathrm{DIV})$ \\
\hline \multirow{2}{*}{$\begin{array}{c}\text { JET } \\
(\mathrm{QH})\end{array}$} & $\approx 15 \mathrm{kHz}$ & $n=1 \sim 6$ & $\tilde{B}_{\theta}, \tilde{n}$ (edge) \\
\hline & (fundamental, EHO) & & \\
\hline \multirow{2}{*}{$\begin{array}{c}\text { AUG } \\
\text { (Type II) }\end{array}$} & Broadband & $n \approx 3-4$ & $\overline{B_{\theta}, \tilde{n} \text { (edge) }}$ \\
\hline & $20-40 \mathrm{kHz}$ & $m \geq 14$ & \\
\hline \multirow{2}{*}{$\begin{array}{c}\text { JET } \\
\text { (Type II) }\end{array}$} & Broadband & $n=8$ & $\tilde{B}_{\theta}, \tilde{n}$ (edge) \\
\hline & $10-30 \mathrm{kHz}$ & & \\
\hline \multirow{2}{*}{$\begin{array}{c}\text { C-Mod } \\
(\text { EDA) }\end{array}$} & $50-150 \mathrm{kHz}$ & $\begin{array}{c}k_{\theta}=1.5-5.0 \mathrm{~cm}^{-1} \\
k \approx 15 \mathrm{~cm}^{-1}\end{array}$ & $\overline{B_{\theta}, \tilde{n}, \tilde{T} \text { (edge) }}$ \\
\hline & $\frac{(\mathrm{QCM})}{\sim 50 \mathrm{kHz}(\mathrm{LF})}$ & $\frac{\kappa_{r} \approx 1.5 \mathrm{~cm}}{n=1 . m \sim 4 \pm 1}$ & $\tilde{B}_{\theta} \tilde{n}, \tilde{\phi}$ (edge, SOL) \\
\hline $\begin{array}{c}\text { JFT-2M } \\
\text { (HRS) }\end{array}$ & $\sim 200-450 \mathrm{kHz}(\mathrm{HF})$ & $n \sim 7 \pm 1$ & $\tilde{I}_{s}($ SOL and DIV) \\
\hline
\end{tabular}

plasma matched to C-Mod show similar wavenumbers and mode location. Nevertheless, the behavior of divertor $D_{\alpha}$ signals indicate no clear enhancement together with the appearance of edge fluctuations. In JET, no controlled EDA modes have been achieved in spite of the existence of edge fluctuations. Moreover, multiple harmonics observed in JET are never observed in C-Mod with a matched shape. Recent intermachine experiments performed in C-Mod and JFT-2M clearly show the similarity of global behavior and operational space between EDA and HRS regimes. Nevertheless, characteristics of edge fluctuations are somewhat different under the matched plasma shape with rather similar non-dimensional pedestal parameters except for aspect ratio. Dominant frequencies of edge fluctuations in EDA and HRS were $\sim 140 \mathrm{kHz}(\mathrm{HF}, n \sim 8$ 28 ) and $\sim 350 \mathrm{kHz}(\mathrm{HF}, n \sim 7)$, respectively. These results suggest that frequency range and mode number (wavenumber) can not be exactly scaled even in the dimensionless matched plasma with similar plasma shape.

It is noted that broadband fluctuations observed in the type II ELM regime have some similarity to the edge fluctuations observed in different regimes with high $\delta$ and high $n_{e}$ plasmas. One good example is found in QH-mode on DIII-D [32]. As the plasma triangularity increased by using a double null configuration, core and pedestal density increased above the critical density, where QH-mode phase can not be sustained at lower triangularity. In this phase, the coherent EHO is replaced by broadband, incoherent edge fluctuations with the frequency range of $f<30 \mathrm{kHz}$. Nevertheless, the pedestal and core 
density can be kept constant at a higher level than that in the low triangularity phase. Therefore, observed broadband fluctuations have enough capability to produce sufficient edge particle loss for the plasma density to reach a constant value. A similar phenomena is also observed in C-Mod [28]. In high density and high input power discharges, the QC mode is replaced by low frequency $(f<50 \mathrm{kHz})$ broadband fluctuations together with the appearance of small irregular ELMs with average frequency around $600 \mathrm{~Hz}$ (green points in Figs. 4(a) and (d)). More detailed studies of changes in the characteristics of edge fluctuations as well as the onset condition of typical edge fluctuations observed in each regime are also important.

\section{Edge Stability}

There are many results of MHD stability analysis applied to some small/no ELM regimes to understand the reason for the suppression mechanism of type I ELMs and leading to small/no ELM regimes. As for the grassy ELM regime, MHD stability analyses to compare between type I ELMing plasmas and grassy ELMing plasmas were performed using the ELITE code [13]. A narrower radial distribution of the eigenfunctions of the most unstable mode for a grassy ELMing plasma than for a type I ELMing plasma is qualitatively consistent with the narrow radial extent of the collapse of temperature pedestal in grassy ELMs. Therefore, both type I ELMs and grassy ELMs seem to be driven by the peeling-ballooning mode. Similar change in the width of the eigenfunctions in a high triangularity plasma was obtained for a type II ELM plasma in AUG [33]. However, there are some contradicting observations in type I ELMs in JET and DIII-D high density plasmas, where narrower eigenfunctions are calculated for that plasma with small type I ELMs showing similar affected area. Therefore, more detailed comparisons are required for better understandings. On the other hand, no ELM regimes of QH-mode and EDA regimes show different results of MHD stability analysis. In both regimes, the edge operational point during the no ELM phase is (marginally) stable to peelingballooning modes [32, 34], while EDA regime with small ELMs is unstable.

Importance of the edge current density to suppress large ELMs is pointed out by the comparison between MHD stability analysis and using current ramp experiments. In JT-60U, a current ramp down to reduce the edge current leads to grassy ELMs and a current ramp up leads to type I ELMs [35]. Similar to this, a current ramp up during QH-mode phase quickly leads to type I ELMs [36]. These responses of small/no ELM regimes against edge current modification are in agreement with peeling-ballooning stability theory. However, further understanding is required to understand why the ELM type is different during constant plasma current phase with steady pedestal parameters.

\section{Discussion and Summary}

Some small/no ELM regimes reported in this paper have possibilities as alternative regimes to type I ELMs. However, there are also some uncertainties and issues to 
establish operational scenarios in ITER with small/no ELMs. As can be seen in Table. 2, in terms of the accessibility to operational conditions in non-dimensional parameters, lower collisionality is required in type II ELM, EDA, HRS and type V regimes. In the grassy ELM regime, a relaxation of the requirement of high $q_{95}$ and $\beta_{p}$ and better understanding of effects of toroidal rotation and $l_{i}$ from the view point of the controllability in the burning plasma should be addressed in the future. In the QH-mode regime, the physical meaning of a requirement of CTR-NBI for steady QH phase and a minimum GAP should be understood. Better understanding of onset conditions for the appearance of these fluctuations in QH-mode, type II ELM, EDA and HRS regimes should be addressed in further experiments.

The required condition to enter the small/no ELM regimes in terms of the plasma shape is also important to investigate further, because ITER cannot operate using a double null configuration and $\Delta_{\text {sep }}$ (the distance between the separatrix and the flux surface through the upper X-point at the outer midplane) should be kept larger than $4 \mathrm{~cm}$. So far, a QDN configuration is required both for type II ELMs and for grassy

Table 2. Summary of global characteristics in various small/no ELM regimes. RL in column $\Delta W_{E L M} / W_{\text {ped }}$ means that values in the column show maximum value evaluated from the resolution limit of the diagnostics.

\begin{tabular}{|c|c|c|c|c|c|}
\hline $\begin{array}{c}\text { Type } \\
\text { (Device) } \\
\end{array}$ & $\begin{array}{c}\text { ELM size } \\
\left(\Delta W_{E L M} / W_{p e d}\right) \\
\end{array}$ & $\begin{array}{c}\text { edge } \\
\text { fluctuaions }\end{array}$ & collisionality & $\begin{array}{l}\text { confinement } \\
\text { property }\end{array}$ & $\begin{array}{c}\text { operational } \\
\text { conditions }\end{array}$ \\
\hline \multirow{2}{*}{$\begin{array}{l}\text { Type II } \\
\text { (AUG) }\end{array}$} & & & & & \\
\hline & $<2 \pm 1 \%(\mathrm{RL})$ & $\overline{\mathrm{BB}}$ & $1.3<\nu_{e}^{*}<3.4$ & $\begin{array}{l}H_{89 P L} \leq 2 \\
H_{98, y 2} \leq 1\end{array}$ & $\begin{array}{c}0.85<\overline{n_{e}} / n_{G W}<0.95 \\
q_{95}>3.5-4 \\
\mathrm{QDN}(\delta \sim 0.4)\end{array}$ \\
\hline (JET) & $<4-5 \%(\mathrm{RL})$ & $\mathrm{BB}$ & $0.8<\nu_{e}^{*}<1.2$ & $H_{98, y 2} \leq 1$ & $\begin{array}{c}n_{\text {ped }} / n_{G W} \geq 0.7 \\
\delta>0.4\end{array}$ \\
\hline \multirow{2}{*}{$\begin{array}{c}\text { Grassy } \\
\text { (JT-60U) }\end{array}$} & & & & & \multirow{4}{*}{$\begin{array}{c}\delta \geq 0.4 \\
q_{95} \geq 4(\text { at } \delta \geq 0.6) \\
\beta_{p} \geq 1.4\end{array}$} \\
\hline & $<1 \%$ & none & $0.2<\nu_{e}^{*}<0.8$ & $\begin{array}{c}H_{89 P L} \leq 2.7 \\
H_{98, y 2} \leq 1\end{array}$ & \\
\hline \multirow{2}{*}{$\begin{array}{l}(\mathrm{JET}) \\
(\mathrm{AUG})\end{array}$} & $<4-5 \%(\mathrm{RL})$ & none & $1.2<\nu_{e}^{*}<1.9$ & $H_{98, y 2} \sim 1.1$ & \\
\hline & $<2 \pm 1 \%(\mathrm{RL})$ & none & $\nu_{e}^{*} \sim 0.8$ & $\begin{array}{c}H_{89 P L} \leq 1.8 \\
H_{98, y 2} \leq 1\end{array}$ & \\
\hline \multirow{5}{*}{$\begin{array}{c}\mathrm{QH} \\
(\mathrm{DIII}-\mathrm{D}) \\
(\mathrm{AUG}) \\
(\mathrm{JT}-60 \mathrm{U}) \\
(\mathrm{JET})\end{array}$} & & & & & \multirow{5}{*}{$\begin{array}{c}\text { CTR-NBI } \\
\text { large GAP } \\
\overline{n_{e}} / n_{G W}<0.47 \\
q_{95} \geq 3.3\end{array}$} \\
\hline & no ELM & EHO & $0.04<\nu_{e}^{*}<0.3$ & $H_{89 P L} \leq 2.2$ & \\
\hline & no ELM & $\mathrm{EHO}+\mathrm{HFO}$ & $\nu_{e}^{*} \sim 0.3$ & $H_{89 P L} \leq 1.6$ & \\
\hline & no ELM & EHO & $\frac{e}{0.4<\nu_{e}^{*}<0.7}$ & $H_{89 P L} \leq 1.7$ & \\
\hline & no ELM & $\mathrm{EHO}$ & & $H_{98, y 2} \sim 1$ & \\
\hline $\begin{array}{c}\text { EDA } \\
\text { (C-Mod) }\end{array}$ & no ELM & QCM & $\nu_{e}^{*} \geq 2$ & $H_{89 P L} \sim 1.9$ & $\begin{array}{c}\text { boronization } \\
q_{95}>3.2, \delta>0.35\end{array}$ \\
\hline $\begin{array}{c}\text { HRS } \\
\text { (JFT-2M) }\end{array}$ & $\begin{array}{c}\text { no ELM } \\
<0.1 \%(\mathrm{RL})\end{array}$ & $\mathrm{LF}+\mathrm{HF}$ & $\nu_{e}^{*} \geq 2$ & $H_{89 P L} \leq 1.6$ & $\begin{array}{c}\text { boronization } \\
q_{95} \geq 3, \delta>0.35\end{array}$ \\
\hline $\begin{array}{l}\text { Type V } \\
\text { (NSTX) }\end{array}$ & $<4.5 \%(\mathrm{RL})$ & none & $\nu_{e}^{*} \geq 2$ & $H_{97 L} \sim 2.2$ & LSN $($ lower $\kappa)$ \\
\hline
\end{tabular}




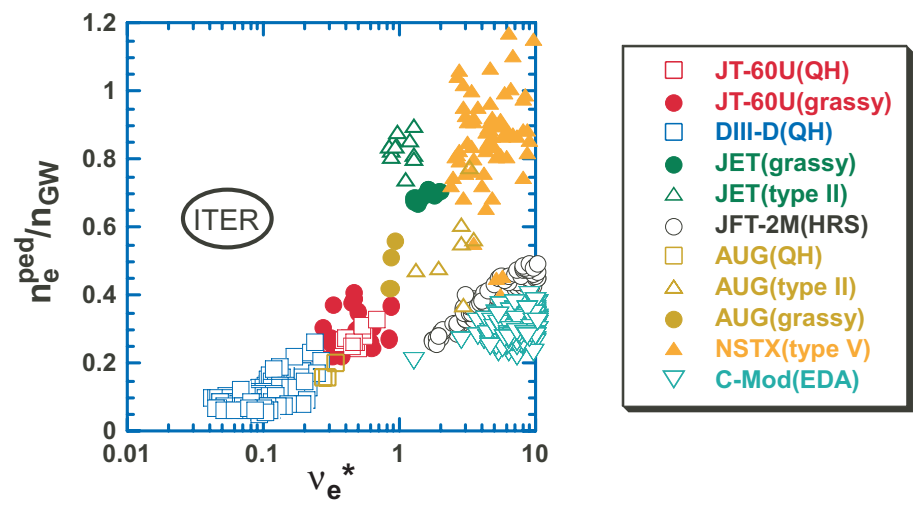

Figure 5. Operational space in $\nu_{e}^{*}$ v.s. $n_{e}^{p e d} / n_{G W}$ for small/no ELM regimes and the type I ELM regime. Different colors show different devices. Open symbols show small/no ELM regimes with edge fluctuations.

ELMs in AUG $[7,12]$. In JET, type II ELM does not require QDN configuration, while grassy ELM has been observed in QDN configuration so far [11]. Grassy ELMs in JT$60 \mathrm{U}$ have often been observed for lower single null (LSN) operation without a second separatrix and type V ELM in NSTX also requires LSN configuration. As noted in the previous section, higher $\delta$ is one important condition for small ELM regimes. Since it is difficult to separate between effects of $\delta$ and $\Delta_{\text {sep }}$ in some devices due to the hardware limitations, we should consider these issues in further experiments.

Another issue is that it is difficult to achieve all non-dimensional parameters matched to ITER plasmas simultaneously. As an example, let us consider the operational space of small/no ELM regimes in $\nu_{e}^{*}-n_{p e d} / n_{G W}$ space as shown in Fig. 5. Collisionality is one important parameter to change the edge stability through the modification of the edge bootstrap current density, while the Greenwald density limit $\left(n_{G W}=I_{p} / \pi a^{2}\right)$ [37] is another important parameter to discuss the density range for ITER, where line-averaged density is expected as $85 \%$ of $n_{G W}$. As can be seen in Fig. 5 , there is an upper boundary in operational points because of the coupling between pedestal density and collisionality due to limited plasma current of present devices. Some small/no ELM regimes show some upper limit of operating pedestal density normalized to $n_{G W}$, but edge collisionality is also changed simultaneously, as can be seen in Fig. 5. It is not so easy to break this coupling using current devices. Under those conditions, investigations of critical parameters leading to small/no ELM regimes among coupled plasma parameters are also encouraged together with the understanding of the suppression mechanism of type I ELMs.

In contrast to the type I ELM regime, small/no ELM regimes reported in this paper are observed under limited pedestal conditions depending on each device. To establish an alternative to type I ELM regimes, continuous efforts to expand the operational regime as well as to establish a robust operational recipe easily applicable to other devices are still required. Since all non-dimensional parameters in ITER cannot be satisfied using current devices as discussed above, further development of a theoretical model 
for small/no ELM regimes is important for confidence in terms of the compatibility to ITER plasmas.

\section{Acknowledgments}

This research was partly supported by the Grant-in-Aid for Scientific Research (S) 17106013, Japan Society for the Promotion of Science.

\section{References}

[1] Federici G, Loarte A and Strohmayer G 2003 Plasma Phys. Control. Fusion 451523.

[2] Shimada M et al 2004 Nucl. Fusion 44350.

[3] Fujita T 2002 Plasma Phys. Control. Fusion 44 A19.

[4] Gohil P 2002 Plasma Phys. Control. Fusion 44 A37.

[5] Sauter O et al 1999 Phys. Plasmas 62834.

[6] Ozeki T et al 1990 Nucl. Fusion 301425.

[7] Stober J et al 2001 Nucl. Fusion 411123.

[8] Saibene G et al 2002 Plasma Phys. Control. Fusion 441769.

[9] Kamada Y et al 2000 Plasma Phys. Control. Fusion 42 A247.

[10] Kamada Y et al 2002 Plasma Phys. Control. Fusion 44 A279.

[11] Saibene G et al 2005 Nucl. Fusion 45297.

[12] Stober J et al 2005 to appear in Nucl. Fusion.

[13] Oyama N et al 2005 Nucl. Fusion 45871.

[14] Saarelma S and Günter S 2004 Plasma Phys. Control. Fusion 461259.

[15] Burrell K.H. et al 2002 Plasma Phys. Control. Fusion 44 A253.

[16] Suttrop W et al 2003 Plasma Phys. Control. Fusion 451399.

[17] Sakamoto Y et al 2004 Plasma Phys. Control. Fusion 46 A299.

[18] Suttrop W et al 2005 Nucl. Fusion 45721.

[19] Zeng L et al 2004 Plasma Phys. Control. Fusion 46 A121.

[20] Suttrop W et al 2004 Plasma Phys. Control. Fusion 46 A151.

[21] Conway G.D. et al. 2004 Plasma Phys. Control. Fusion 46951.

[22] Burrell K.H. et al 2004 Plasma Phys. Control. Fusion 46 A165.

[23] Greenwald M et al 1997 Nucl. Fusion 37793.

[24] Kamiya K et al 2003 Nucl. Fusion 431214.

[25] Maingi R et al 2005 Nucl. Fusion 45264.

[26] Kamiya K, Oyama N, Miura Y and Hubbard A.E. 2004 Plasma Phys. Control. Fusion 461745.

[27] Maingi R et al 2005 Nucl. Fusion 451066.

[28] Mossessian D.A. et al 2003 Phys. Plasmas 101720.

[29] Kamiya K et al 2004 Proc. 20th Int. Conf. on Plasma Physics and Controlled Nuclear Fusion Research IAEA-CN-116/EX/4-6Ra.

[30] Mossessian D.A. et al 2003 Phys. Plasmas 10689.

[31] Hubbard A.E. et al 2005, in this conference, "Comparisons of small ELM H-mode regimes on the Alcator C-Mod and JFT-2M tokamaks".

[32] Burrell K.H. et al 2005 Phys. Plasmas 12056121.

[33] Saarelma S et al 2003 Nucl. Fusion 43262.

[34] Mossessian D.A. et al 2002 Plasma Phys. Control. Fusion 44423.

[35] Oikawa $\mathrm{T}$ et al 2002 "Comparison of large and small ELM regimes in JT-60U and DIII-D", at 44 th Mtg of APS Div. of Plasma Physics, Orlando.

[36] West W.P. et al 2004 Plasma Phys. Control. Fusion 46 A179.

[37] Greenward M et al 1988 Nucl. Fusion 282199. 